\title{
THURSTON NORM MINIMIZING SURFACES AND SKEIN TREES FOR LINKS IN $S^{3}$
}

\author{
ABIGAIL THOMPSON \\ (Communicated by Frederick R. Cohen)
}

\begin{abstract}
This paper gives a method for constructing all links in $S^{3}$, beginning with the unknot and adding at most one to the norm of the link at each stage. This has two corollaries. The first is that links with 'minimal' skein trees are fibered. The second is a complete list of all links with skein trees of height two.
\end{abstract}

In [2] it is shown that three links related by the Conway moves have closely related Thurston norm minimizing Seifert surfaces. This is used to give a lower bound on the height of a skein tree for a link. Here these results are extended to yield a method for constructing all links, beginning with the unknot and adding at most one to the norm of the link at each stage. This has two corollaries: the first is that if the lower bound obtained in [2] is realized, then the link must be fibered. The second is a complete list of all links with skein trees of height two. It is surprisingly easy to obtain this second corollary, especially when one considers that the question of characterizing skein trees of height one is equivalent to the question of whether one can band together two knots in some non-trivial way to obtain the unknot. This was a long-standing problem, eventually solved by M. Scharlemann [1].

(1.1) Definition. A Seifert surface for an oriented link $L$ in $S^{3}$ is an oriented surface $S$, with no closed components, such that $\partial S=L . S$ is taut if there is no Seifert surface for $L$ of larger Euler characteristic. This is equivalent to being Thurston norm minimizing and incompressible [2]. Let $X(L)$ be minus the Euler characteristic of a taut Seifert surface for $L$.

(1.2) Definition. Three oriented links $L_{+}, L_{-}$and $L_{0}$ in $S^{3}$ are related by the Conway moves if they are identical outside a three-ball $B$ where they appear as in Figure 1. We refer to [2] for the definitions of a skein tree $T$ for a link $L$, a node of $T$, a leaf of $T$, the height of a skein tree for $L$ and the height of $L$, written $h(L)$.

Received by the editors August 25, 1988.

1980 Mathematics Subject Classification (1985 Revision). Primary 57M25.

The author was supported in part by a National Science Foundation grant and the Lady Davis Fellowship Trust. 

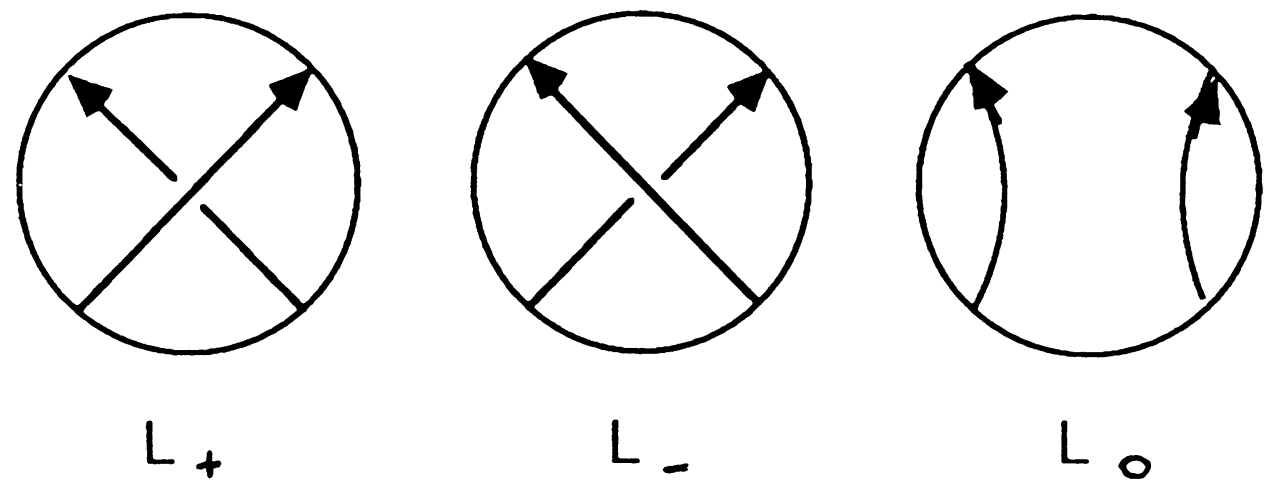

FIGURE 1

(1.3) Definition. Let $L=L_{+(-)}$be a node of a skein tree $T$, and let $L_{-(+)}$ and $L_{0}$ be the links in $T$ obtained from $L_{+(-)}$by the Conway moves. Call $L_{-(+)}$the left branch of $L_{+(-)}$and $L_{0}$ the right branch.

(1.4) Definition. Let $S$ be a Seifert surface for the link $L, \alpha$ an arc properly imbedded in $S . L^{\prime}$ is obtained from $L$ by twisting along $\alpha$ if $L$ and $L^{\prime}$ are related as shown in Figure $2 \mathrm{a} . L^{\prime}$ is obtained from $L$ by adding a twisted band along $\alpha$ if $L$ and $L^{\prime}$ are related as shown in Figure 2b. Note that the operation of adding a twisted band along an arc is the same as plumbing a Hopf band to $S$ along $\alpha$ (see [3]). Also note that both operations yield an obvious Seifert surface $S^{\prime}$ for $L^{\prime}$.

(1.5) Definition. A link $L$ in $S^{3}$ is split if it contains an essential 2-sphere in its complement. Otherwise it is non-split.

(1.6) Theorem. Let $L$ be a non-split link in $S^{3}$. Then there exists a sequence of triples $\left(L_{0}, S_{0}, \alpha_{0}\right) \rightarrow\left(L_{1}, S_{1}, \alpha_{1}\right) \rightarrow \cdots \rightarrow\left(L_{m-1}, S_{m-1}, \alpha_{m-1}\right) \rightarrow L_{m}=L$ such that:

1. $L_{0}$ is the unknot.

2. $m \leq h(L)$.

3. $S_{i}$ is a taut Seifert surface for $L_{i}, i=0, \ldots, m-1$.

4. $\alpha_{i}$ is an arc properly imbedded in $S_{i}$.

5. $L_{i+1}$ is obtained from $L_{i}$ by either twisting along $\alpha$ or by adding $a$ twisted band along $\alpha$.

Proof. Let $T$ be a skein tree for $L$ of height $h(L)$. We choose an imbedded path $p$ in $T$ from $L$ to a leaf of $T$ as follows: suppose $L_{+(-)}$is a node of $T$, with left branch $L_{-(+)}$and right branch $L_{0}$. If $X\left(L_{-(+)}\right) \geq X\left(L_{+(-)}\right)$, connect $L_{+(-)}$to $L_{-(+)}$. Otherwise connect $L_{+(-)}$to $L_{0}$. Beginning with $L$, use this 
a)

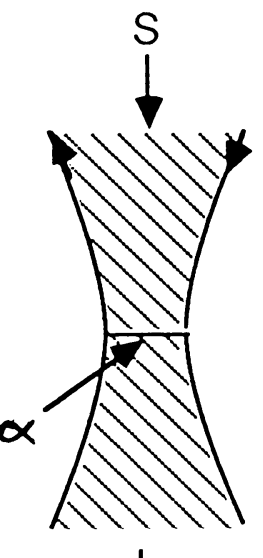

$\mathrm{L}$
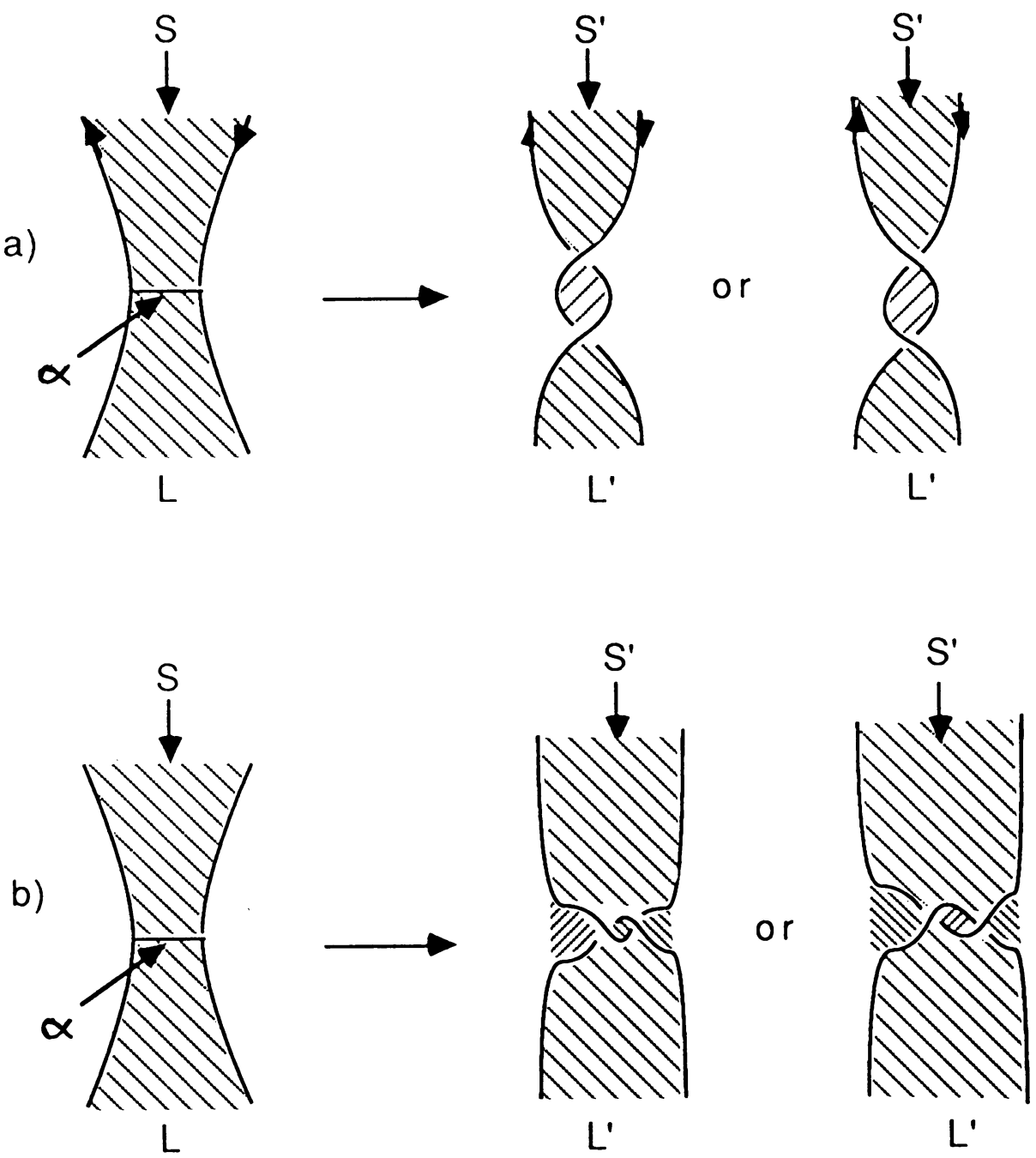

FIGURE 2

rule to construct $p$. Label the links in $p$ from zero to $m$, beginning with the leaf of $T$. Note that $m \leq h(L)$.

Suppose $L_{i}$ is a right branch of $L_{i+1}$. Then $X$ (left branch $\left.L_{i+1}\right)<X\left(L_{i+1}\right)$. By [2, Proposition 3.1], it follows that there exists a taut Seifert surface $S_{i}$ for $L_{i}$ and an arc $\alpha_{i}$ properly imbedded in $S_{i}$ such that $L_{i+1}$ can be obtained from $L_{i}$ by adding a twisted band along $\alpha_{i}$.

Suppose $L_{i}$ is a left branch of $L_{i+1}$. Then $X\left(L_{i}\right) \geq X\left(L_{i+1}\right)$. Using the arguments from [2, Theorem 1.4], it follows that there exists a taut Seifert surface $S_{i}$ for $L_{i}$ and an arc $\alpha_{i}$ properly imbedded in $S_{i}$ such that $L_{i+1}$ can be obtained from $L_{i}$ by twisting along $\alpha_{i}$. 
It remains to show that $L_{0}$ is indeed the unknot as opposed to an unlink of $j>1$ components.

Claim. Let $L$ be a split link in $S^{3}$. If $S$ is a taut Seifert surface for $L$, then one can find a splitting sphere $F$ such that $S \cap F=\varnothing$.

Proof. Choose $F$ to minimize the number of components in $S \cap F$. If $S \cap F \neq$ $\varnothing$, then an innermost disk argument shows that $S$ is compressible, hence not taut.

From this claim it follows that if $L_{i}$ in the above construction is split, then so is $L_{i+1}, i=0, \ldots, m-1$. Since we assume that $L$ is not a split link, it follows that $L_{0}$ is not split, hence $L_{0}$ is the unknot.

(1.7) Corollary. If $L$ is a non-split link in $S^{3}$ such that $h(L)=X(L)+1$, then $L$ is a fibered link.

Proof. Let $L$ be a link in $S^{3}$ with $h(L)=n$ and let

$$
\left(L_{0}, S_{0}, \alpha_{0}\right) \rightarrow\left(L_{1}, S_{1}, \alpha_{1}\right) \rightarrow \cdots \rightarrow\left(L_{m-1}, S_{m-1}, \alpha_{m-1}\right) \rightarrow L_{m}=L
$$

be a sequence of triples for $L$ constructed as in Theorem 1.6.

Claim. Then $X(L) \leq m-1$, and equality holds if and only if $L_{i+1}$ is obtained from $L_{i}$ by adding a twisted band along $\alpha_{i}$ for all $i=0, \ldots, m-1$.

Proof. In the proof of 1.6, $L_{i+1}$ is obtained from $L_{i}$ by twisting along $\alpha_{i}$ if and only if $X\left(L_{i+1}\right) \leq X\left(L_{i}\right)$. So also we know that if $L_{i+1}$ is obtained from $L_{i}$ by adding a twisted band along $\alpha_{i}, X\left(L_{i+1}\right)>X\left(L_{i}\right)$. But the Seifert surface $S^{\prime}$ constructed for $L_{i+1}$ by plumbing a Hopf band to $S_{i}$ along $\alpha_{i}$ has $-\chi\left(S^{\prime}\right)=-\chi\left(S_{i}\right)+1=X\left(L_{i}\right)+1$, so $X\left(L_{i+1}\right) \leq X\left(L_{i}\right)+1$. Hence $X\left(L_{i+1}\right)=X\left(L_{i}\right)+1$. Since $X\left(L_{0}\right)=-1$, it follows that $X(L) \leq m-1$, with equality if and only if $L_{i+1}$ is obtained from $L_{i}$ by adding a twisted band along $\alpha_{i}$ for all $i=0, \ldots, m-1$.

Hence we know $X(L)+1 \leq m \leq n$. If $X(L)+1=h(L)$, then $m=h(L)$ and $L_{i+1}$ is always obtained from $L_{i}$ by adding a twisted band along $\alpha_{i}$ for $i=0, \ldots, m-1(=h(L)-1)$. Plumbing a Hopf band to the taut Seifert surface of a fibered knot yields a fibered knot [3], hence $L$ is fibered.

(1.8) Corollary. The following is a complete list of all non-split links of height less than or equal to two (see Figure 3); it is obvious from the proof that there are some restrictions on the orientations of the components in 5). These are indicated in Figure 3.

1. the unknot $(h=0)$;

2. the Hopf links $(h=1)$;

3. the right- and left-handed trefoils;

4. the figure eight knot;

5. the Hopf link with an extra twist;

6. a chain of three unknotted components. 
1)

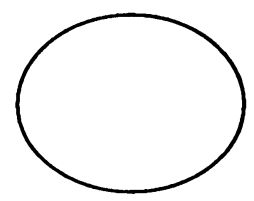

3)

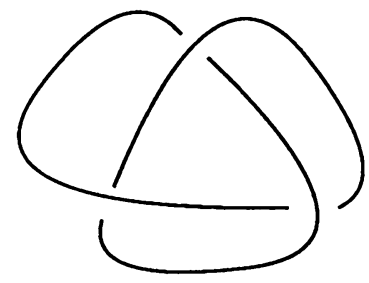

4)

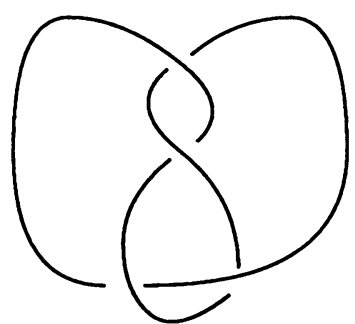

5)

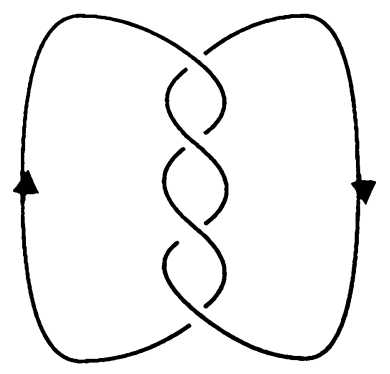

6)

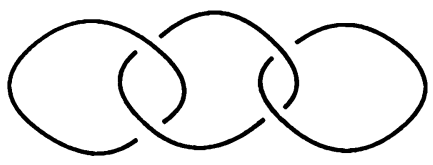

2)
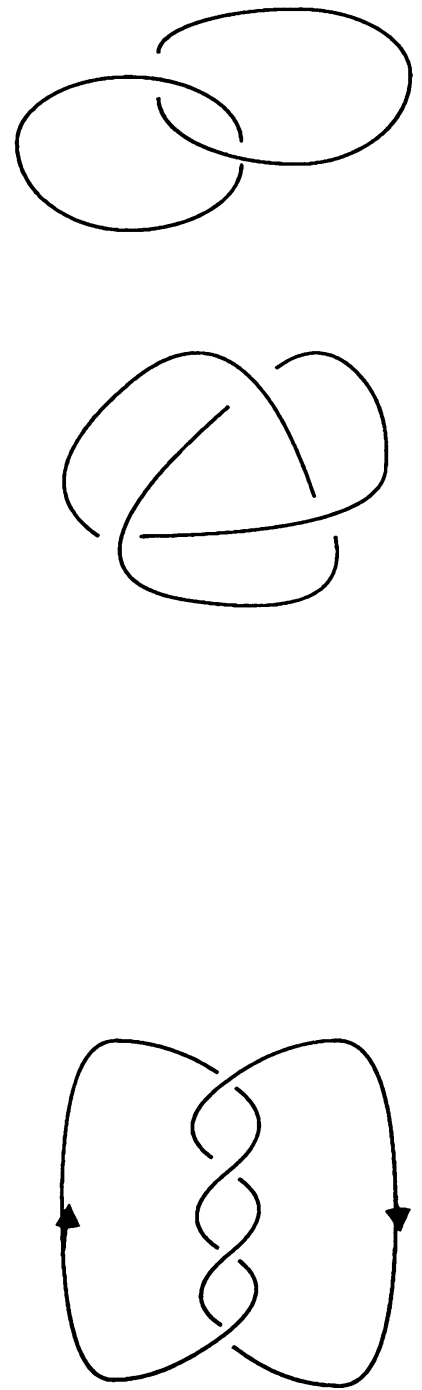

FIGURE 3

Proof. Suppose $h(L) \leq 2$. Apply Theorem 1.6 to obtain a sequence of at most two triples. By the theorem, $L_{0}$ is the unknot. If we twist along $\alpha_{0}$, we obtain $L_{1}$ also equal to the unknot, so without loss of generality we can assume that at the first stage we add a twisted band along $\alpha_{0}$. If we add a twisted band along $\alpha_{0}$ we obtain $L_{1}$ equal to the Hopf link (2). A taut Seifert surface for the Hopf link is an annulus $A$, unique up to isotopy. The annulus contains only two distinct arcs up to automorphism, one essential and one inessential. 
Case (i). Suppose $\alpha_{1}$ is an essential arc in $A$. If we add a twisted band along $\alpha_{1}$ we obtain either one of the two trefoils (3) or the figure eight knot (4). If we twist along $\alpha_{1}$ we obtain either the trivial link of two components, which is split, or the Hopf link with an extra twist (5).

Case (ii). Suppose $\alpha_{1}$ is not essential in $A$. If we twist along $\alpha_{1}$ we again obtain the Hopf link. If we add a twisted band along $\alpha_{1}$ we obtain a chain of three unknotted components (6).

This shows that 1-6 are the only non-split links that could possibly have height less than or equal to two. It remains to show that these links indeed all have skein trees of height less than or equal to two. One can easily construct such trees for these links; this is left to the reader.

\section{ACKNOWLEDGMENT}

I would like to thank Marty Scharlemann for many helpful conversations.

\section{REFERENCES}

1. M. Scharlemann, Smooth spheres in $\mathbf{R}^{4}$ with four critical points are standard, Invent. Math., 79 (1985), 125-141.

2. M. Scharlemann and A. Thompson, Link genus and the Conway moves, to appear in Comm. Math. Helv.

3. J. Stallings, Constructions of fibered knots and links, A.M.S. Proc. Symp. Pure Math., 32 (1978), 55-60.

Department of Mathematics, University of California, Davis, California 95616 